\title{
Numerical optimization of writing computer-generated holograms in threshold media
}

\author{
Mateusz Sadowski, Michał Makowski* \\ Faculty of Physics, Warsaw University of Technology, 75 Koszykowa, 00-662 Warsaw, Poland
}

Received August 03, 2021; accepted November 13, 2021; published December 31, 2021

\begin{abstract}
Recent developments in binary photo-magnetic materials showed efficient and ultra-fast rewriting of holograms where the intensity threshold allows for a dense, sub-diffraction limit packing of hologram points. This paper describes the numerical optimization of the process of writing and reconstructing of 2-D images in a binary-phase computer-generated holograms stored in the said threshold-like medium. Global free parameters optimization of the writing process is shown, including the intensity threshold level, propagation distance, hologram spot size and the shape of the boundary regions of the written spots. We present the optimal set of parameters for the best possible writing quality.
\end{abstract}

Computer-generated holography (CGH) allows realtime design, writing and playback of phase-only or amplitude-only holograms. Usually it is realized by computer-aided design, connected with the frame-byframe display on a spatial light modulator (SLM), most likely based on the liquid-crystal-on-silicon (LCoS) technology. Although this scheme is well known and widely used, some intrinsic limitations of LC-based displays, like slow refresh rates and lack of internal memory, slow down the progress in $\mathrm{CGH}$ development for final applications in holographic television of the future. For those reasons, novel and faster materials for dynamic $\mathrm{CGH}$ are being developed. Among them are photo-magnetic materials capable of light-induced patterning at $10 \mathrm{GHz}$ rates with femtosecond laser pulses $[1,2]$. The area of switched magnetic domains in this process is related with the intensity and focusing of the fs pulse, since the medium has a certain intensity threshold [3]. For this reason, the CGH design and writing parameters must be optimized for such binary, thresholdlike materials. In this work we present the outcome of such initial optimization, leading to the best possible writing and playback scheme in future experiments.

In this paper, the simulation of diffraction and propagation was done using the Angular Spectrum Method [4]. The test binary-phase iterated [5] hologram presenting a test " 8 " symbol with a rectangle was oversampled by 16 times and rewritten with a threshold. As seen in Fig. 1, the produced pattern was numerically illuminated with a convergent beam focusing at the preset propagation distance. This numerical experiment

\footnotetext{
*E-mail: michal.makowski@pw.edu.pl
}

clearly resembles a typical configuration of the holographic projection of 2-D images $[6,7]$.

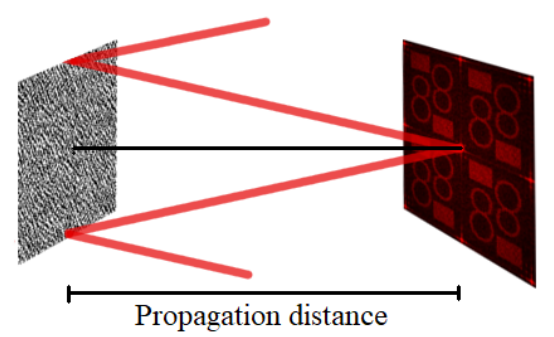

Fig. 1. Simulating the propagation of a holographic pattern illuminated with a convergent beam.

Each sample (pixel) of the input holographic pattern was rewritten into a square cell of 16 by 16 pixels, as seen in Fig. 3. This oversampling has allowed us to simulate the threshold writing by binarization of a Gaussian beam pattern within the cell, as depicted by the green line in Fig. 2. In a separate test additional gradient samples, called superpixels were added on the transition between 1 and 0 values representing gradual change of intensity. This parameter potentially allows one to investigate the fine differences in the performance of materials with different profiles of boundaries between written and notwritten domains, which often depends on the layer thickness [1].

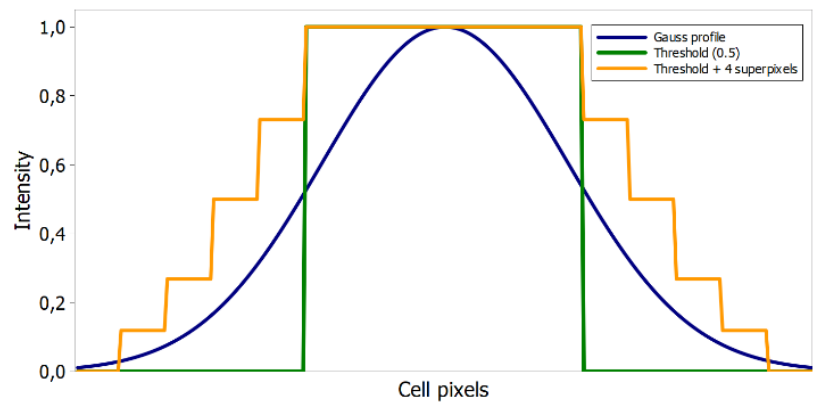

Fig. 2. Comparison of intensity in oversampled representations of the elementary cell of the hologram. 


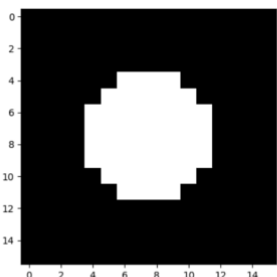

Cell with threshold writing

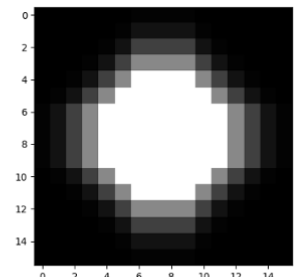

Cell with threshold writing and 4 superpixels
Fig. 3. Examples of hologram cells with gradient superpixels.

Although the simulation has many variables which can be changed the analysis and optimization of parameters has been done only for values most impactful on the quality of the reconstructed image. The tested parameters were: • Propagation distance, • Value of threshold in cell writing, - Width of the writing gaussian beam, - Size of the pattern, $\bullet$ Number of superpixels.

The base parameters were used for each of the simulations:

- Wavelength: $632.8 \mathrm{~nm}$,

- Pattern size: $30 \times 30 \mathrm{~mm}$,

- Propagation / screen distance: $1 \mathrm{~m}$,

- Oversampling: $16 \times 16$,

- Superpixels: 0

- Threshold value: 0.5 ,

- Gaussian beam width: $0.08 \mathrm{~mm}$.

In Fig. 4 examplary far field reconstructions of the test hologram for different sets of the simulation parameters are presented. Plot $A$ represents the default parameters of the simulation. Plot $B$ shows the changes due to compressing the pattern into a $15 \times 15 \mathrm{~mm}$ square, expanding the output image. Plot $C$ is visibly dimmer and slightly larger thanks to $2.5 x$ larger propagation distance. Plot D displays the change in intensity distribution caused by 4 additional superpixels in cells of the pattern.

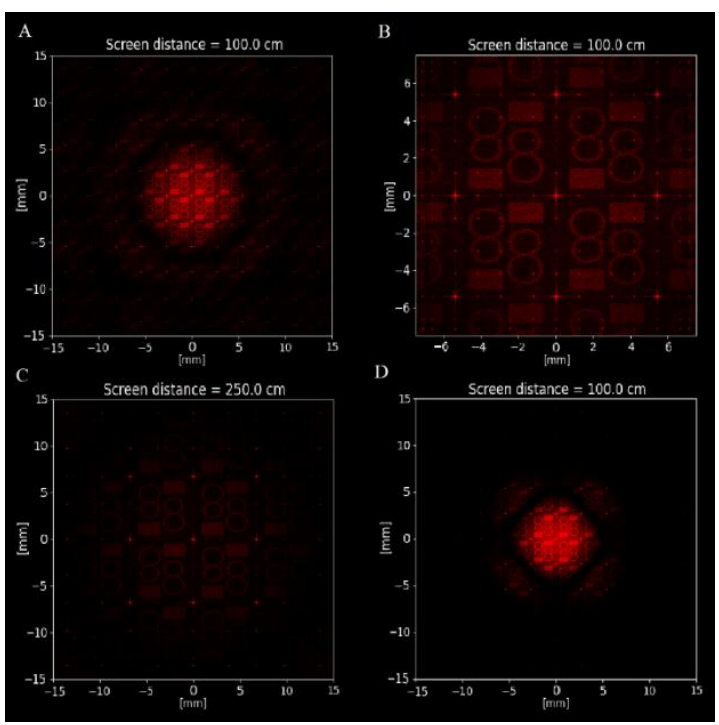

Fig. 4. Examples of far field intensity reconstructions for different sets of simulation variables.
Parameters of the reconstructed images in each simulation series were statistically analysed from each obtained numerical far field playback. Diffraction efficiency in $1^{\text {st }}$ order, contrast and speckle noise were calculated after separating the image from the background using filtering and then combined into a joint statistic quality $(Q)$ value representing the weighted performance of the playback. The $Q$ value is defined as follows:

$$
Q=\frac{100 * \text { D.Eff. }+0.5 * \text { Cont } .+2.5 * \frac{1}{\text { Speckle Noise }}}{4}
$$

Figures 5-9 contain graphs showing the results of tested relations. Right side graphs present the $Q$ statistics while on the left there are presented separated data points representing the normalized diffraction efficiency, contrast, and speckle noise (brown, green and blue circles, respectively) for each series. Value of 1 means the best achieved result in the graphs on the left.

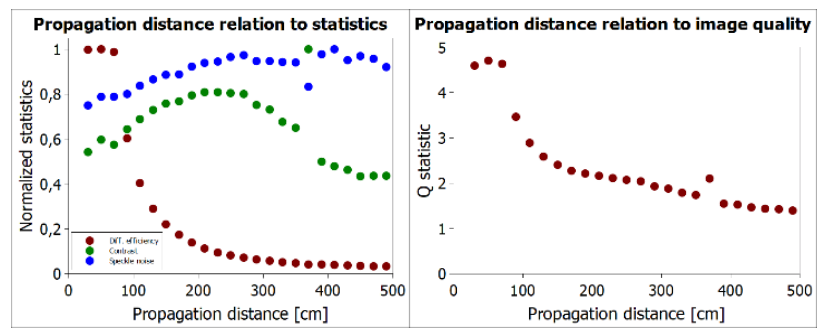

Fig. 5. Influence of the propagation distance on the image statistics.

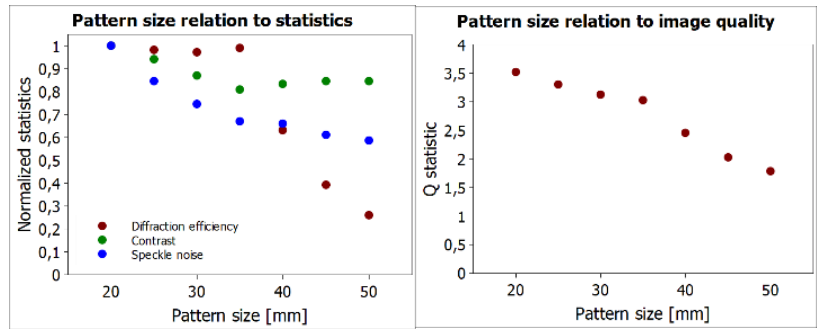

Fig. 6. Influence of the hologram size on the image statistics.

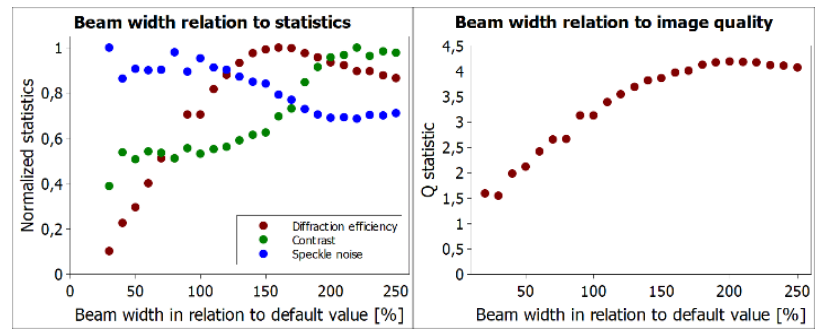

Fig. 7. Influence of the writing beam width on the image statistics. 


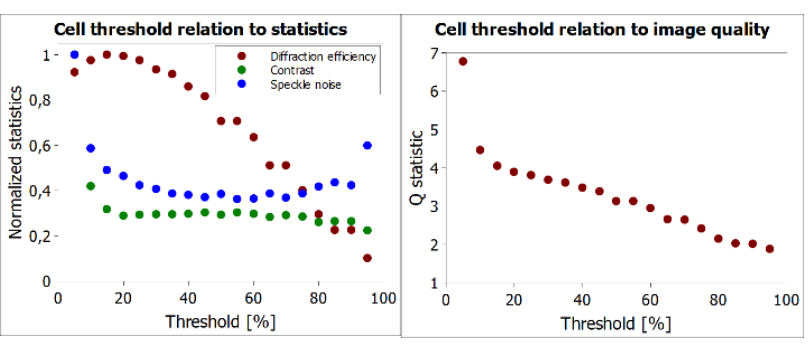

Fig. 8. Influence of the threshold value on the image statistics.

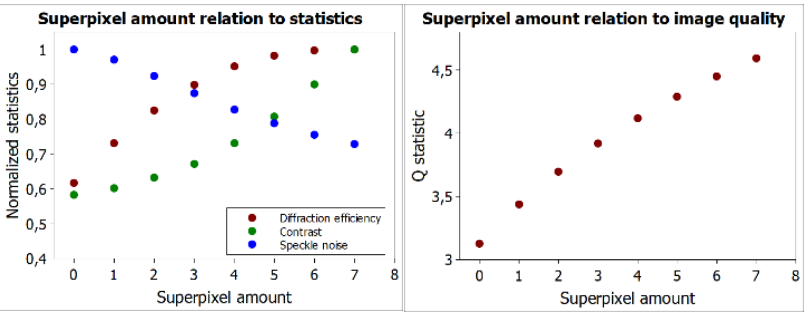

Fig. 9. Influence of the superpixel count on the image statistics.

Due to threshold and beam width parameters being linked the conclusion from our results is that the cells of the pattern should be filled as much as possible while remaining circular. Superpixels can be used to fill the remaining gaps. Smaller patterns have better diffraction efficiency and speckle noise. Closer propagation allows for better diffraction efficiency but makes the image less clear because of the $0^{\text {th }}$ order of diffraction.

Using the changes, we have achieved a statistically optimized result shown in Fig. 10. Plot A represents the default parameters. Plot B uses the optimized parameters allowing it to achieve a higher diffraction efficiency and less noise while sacrificing some contrast due to the $0^{\text {th }}$ order. Plot $\mathrm{C}$ shows simulation using the same parameters at further distance achieving higher contrast at the cost of the other statistics. The elementary hologram cell formed from the optimal set of parameters is shown in Fig. 11.

We have shown that the optimization of the holographic recording in a threshold-like medium requires a careful selection of parameters of the writing beam. No global optimum was found to satisfy all quality measures. Based on the preselection of the most critical quality measure in a given experiment, the optimal set of beam properties can be taken based on the graphs presented here. The fine adjustment of the parameters of the process allows for obtaining a better and clearer image with superior diffractive efficiency, which is significant for future holographic experiments on exotic materials exhibiting the intensity threshold.

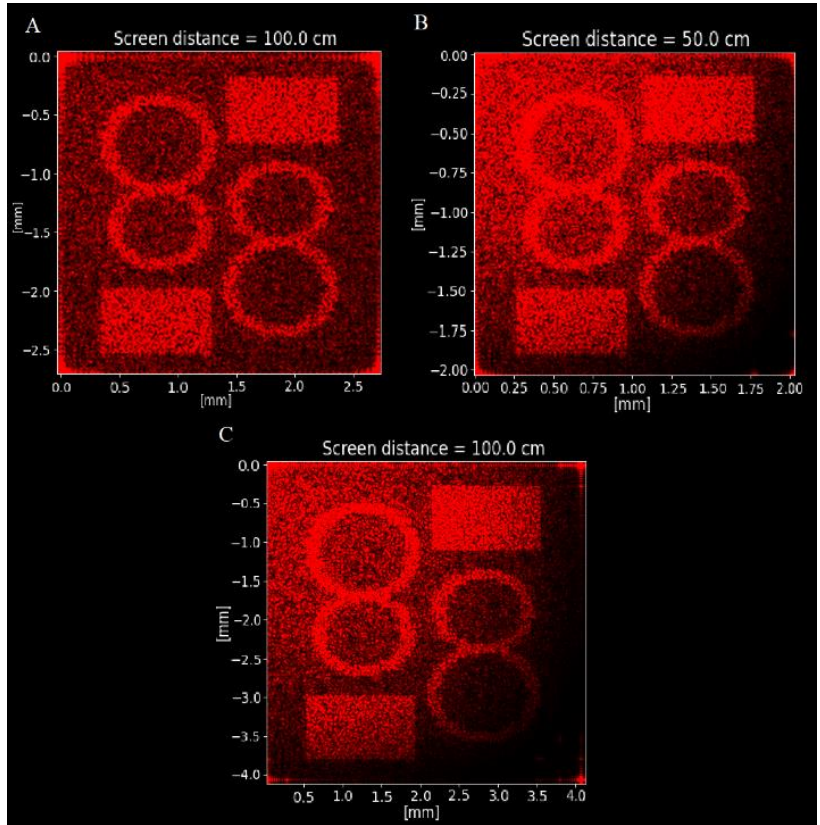

Fig. 10. Far field reconstructions with default and optimized parameters.

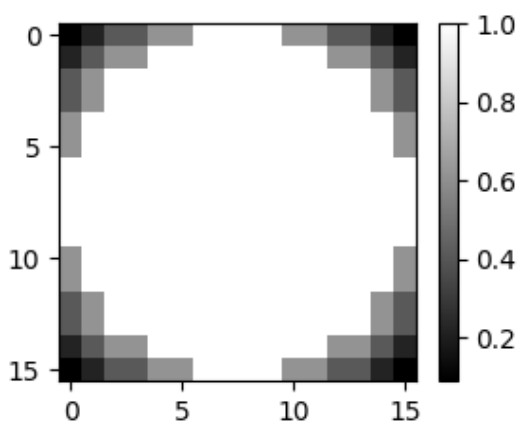

Fig. 11. The optimal configuration of the elementary writing cell of the hologram.

\section{References}

[1] A. Stupakiewicz, K. Szerenos, D. Afanasiev et al., Nature 542, 71 (2017).

[2] J. Starobrat, A. Frej, J. Bolek, R. Trybus, A. Stupakiewicz, M. Makowski, Opt. Lett. 45, 5177 (2020).

[3] V. Ostroverkhov et al., Jap. J. App. Phys. 48.3S1, 03A035 (2009)

[4] K. Matsushima, T. Shimobaba, Opt. Express 17, 19662 (2009).

[5] F. Wyrowski, O. Bryngdahl, JOSA A 5.7, 1058 (1988).

[6] I. Ducin, T. Shimobaba, M. Makowski, K. Kakarenko, A. Kowalczyk, Jaroslaw Suszek, M. Bieda, A. Kolodziejczyk, M. Sypek, Opt. Comm. 340, 131 (2015).

[7] M. Makowski, Opt. Express 21, 29205 (2013). 